\title{
Development the nuclear decay data sublibrary for fission product
}

\author{
Huang Xiaolong ${ }^{1}$, Liu Lile ${ }^{1}$, Yang Dong ${ }^{2}$, Li Jian ${ }^{2}$ \\ ${ }^{1}$ China Nuclear Data Center, China Institute of Atomic Energy, Beijing 102413, China \\ ${ }^{2}$ College of Physics, Jilin University, Changchun 130012, China
}

\begin{abstract}
Accurate and reliable nuclear decay data libraries are essential for calculation and design of the advanced nuclear systems. To meet the requirements of decay heat calculation, burn-up calculation, analysis neutrino spectra anomaly and the other related studies, the evaluated nuclear decay data sublibrary for fission product has been developed and constituted for the first time in China based on the several main national evaluated data libraries in the world. About 1415 nuclides were included in present database. And two major data format, that's ENSDF and ENDF were provided. Some decay heat calculation, reactor neutrino spectra analysis and decay chain design needed in fission yield evaluation were performed to test the accuracy and reliability of the nuclear decay data sublibrary.
\end{abstract}

Keywords: nuclear decay data; sublibrary; fission product;

\section{Introduction}

Evaluated nuclear structure and decay data are widely used in science, industry, medicine and agriculture. The decay data of fission products, actinides and their decay products are extremely important in nuclear applications such as burn-up analysis and decay heat calculation of fission products. To meet these nuclear application needs, the fission product decay data library should be developed. The information from such databases can be widely used in calculations of induced activation, transmutation of isotopes, and heat production.

On the basis of new measurements and some well known evaluated decay data files the decay data of various radionuclides have been evaluated. Recommended decay data characteristics include half-lives, branching fractions, alpha, beta and gamma-ray energies and emission probabilities, total decay energies, mean electromagnetic, light-particle and heavy-particle energies, and all associated uncertainties. All of the evaluated data have been converted into ENDF- 6 format.

In this paper, the brief instructions of status and progress of the decay data sublibrary are presented.

\section{Contents of the decay data library for fission product}

To construct the decay data sublibrary for fission product, the information of well known evaluated decay data files in the world such as ENSDF, DDEP database, and CNDC evaluations were very useful. These evaluated information was compared to the available new measurements and was considered in present database. Some theoretical analyzed results were also included.
The information presented in the decay data sublibrary for fission product was compiled and derived from four sources: 1) ENSDF (http://www.nndc.bnl.gov/ensdf) [2]; 2) DDEP (http://www.nucleide.org/DDEP.htm) [3]; 3) JEFF3.3 (http://www.oecd-nea.org /dbdata/jeff/jeff33/) [4]; 4) CNDC evaluations. All the total decay energies Q-value were updated using the new mass adjustment[1]. The information for betadelayed neutron if the precursor existed were taken from IAEA recommendations [2].

For each nuclide the following decay characteristics are given. The half-lives, branching fractions, alpha, beta and gamma-ray energies and emission probabilities, total decay energies, mean electromagnetic, light-particle and heavyparticle energies, and all associated uncertainties, are included in data file. All evaluated information was compared to the available measurements.

Finally a consistency of evaluated decay scheme was studied. The consistency of the recommended data in all of the decay schemes was determined by comparing the effective Qvalue and the calculated Q-value as below:

$\mathrm{R}=[($ effective Q-value - calculated Q-value $) /$ effective Qvalue $]^{*} 100$

The two values are from two different independent ways. The effective Q-value is taken from the atomic mass data of Wang et al.' work [1]. The calculated Q-values is derived from the summation of the energies taken by all the particles emitted in the decay process, including the alpha or beta (maximum energy), gamma, X-ray and conversion electrons et al. The calculation employed the energies and the emission probabilities of all particles based on the evaluated decay level scheme. Hence, the $\mathrm{R}$ value reflects the correction and the completement of the decay level scheme. 
A value of $\mathrm{R}$ above $5 \%$ are regarded as a poorly decay scheme, whereas less than $5 \%$ indicates the construction of a reasonably decay scheme. For a nuclide with reasonably decay scheme, the total mean beta and gamma energies were calculated from recommended decay data using LOGFT and RADLIST codes [6]. For a nuclide with poorly or no decay scheme, the total mean beta and gamma energies were determined from the TAGS studies of Greenwood et al. [7] when available, otherwise were obtained from the our theoretically analysis which employed the self-consistent quasiparticle random phase approximation (QRPA) approach based on covariant density functional theory (CDFT), which has successfully described nuclear beta-decay half-lives [8,9]. Table 1 gives some examples of even-even nuclides from our theoretically analysis.

Table 1 Examples of calculated results by the QRPA approach comparing with the experimental data [10-12].

\begin{tabular}{|l|c|c|c|c|c|c|}
\hline \multirow{2}{*}{ Nucleus } & \multicolumn{2}{|c|}{$\mathbf{T}_{\mathbf{1} / \mathbf{2}}$} & \multicolumn{2}{c|}{$\mathbf{Q}_{\beta}(\mathbf{k e V})$} & \multicolumn{2}{c|}{$\mathbf{Q}_{\gamma}(\mathbf{k e V})$} \\
\cline { 2 - 7 } & $\mathbf{E x p}^{*}$ & Theo. & Exp. & Theo. & Exp. & Theo. \\
\hline${ }^{88} \mathrm{Kr}^{[10]}$ & $2.825 \mathrm{~h}$ & $3.069 \mathrm{~h}$ & 356 & 349 & 1953 & 1935 \\
\hline${ }^{116} \mathrm{Pd}^{[11]}$ & $11.8 \mathrm{~s}$ & $11.5 \mathrm{~s}$ & 995 & 853 & 155 & 568 \\
\hline${ }^{146} \mathrm{Ce}^{[12]}$ & $13.5 \mathrm{~m}$ & $14.5 \mathrm{~m}$ & 232 & 311 & 305 & 128 \\
\hline
\end{tabular}

\section{The brief description of decay data library}

Two major data format, i.e., ENSDF and ENDF were adopted in present database. After the recommendation of a particular radionuclide was completed, the recommended decay data were prepared and converted into ENDF-6 format. For each nuclide, the recommended decay data for the following characteristics are contained within the data section $(\mathrm{MF}=8$, $\mathrm{MT}=457)$ :

1) half-lives;

2) total decay energies (Q-values);

3) branching fractions, isomeric branching fractions for isomeric state;

4) mean alpha, beta and gamma energies;

5) alpha-particle energies and emission probabilities;

6) beta-particle energies, emission probabilities and transition types;

7) gamma-ray energies, emission probabilities;

8) all associated uncertainties.

\section{Conclusions}

The evaluated nuclear decay data sublibrary for fission product in China has been developed. Recommended decay data have been taken from various sources, mainly from the ENSDF, DDEP database and CNDC evauations. All of the evaluated data have been generated with ENSDF and ENDF data format. The mean $\beta$ and $\gamma$ energies have been deduced from recommended decay data if decay scheme is reasonably, otherwise from total absorption gamma-ray spectroscopy (TAGS) measurements and theoretical calculations. Testing of the decay data sublibrary will be undertaken and released together with new CENDL in future.

\section{References}

1. M. Wang, G. Audi, F. G. Kondev, W. J. Huang, S. Naimi, X. Xu, Chin.Phys.C 41, 030003 (2017)

2. http://www.nndc.bnl.gov/ensdf

3. http://www.nucleide.org/DDEP.htm

4. http://www.oecd-nea.org/dbdata/jeff/jeff33/

5. J. Liang, B. Singh, E.A. McCutchan, I. Dillmann, M. Birch, A.A. Sonzogni, X. Huang, M. Kang, J.Wang, G. Mukherjee, K. Banerjee, D. Abriola, A. Algora, A.A. Chen, T.D. Johnson, and K. Miernik, Nuclear Data Sheets, in press

6. https://www-nds.iaea.org/public/ensdf_pgm

7. R.C. Greenwood, R.G. Helmer, M.H. Putnam, K.D. Watts, Nucl. Instrum. Methods Phys. Res. A390, 95 (1997)

8. Z. M. Niu, Y. F. Niu, Q. Liu, H. Z. Liang, and J. Y. Guo, Phys. Rev. C 87, 051303(R) (2013)

9. Z. M. Niu, Y. F. Niu, H. Z. Liang, W. H. Long, and J. Meng, Phys. Rev. C 95, 044301 (2017)

10. G. Mukherjee, Nuclear Data Sheets 105, 419-556 (2005)

11. Jean Blachot, Nuclear Data Sheets 111, 717-895 (2010)

12. Yu. Khazov, A. Rodionov and G. Shulyak, Nuclear Data Sheets 136, 163-452 (2016) 